\title{
Impact of surveillance among patients with resected pancreatic cancer following adjuvant chemotherapy
}

\author{
Selina K. Wong, Lovedeep Gondara, Daniel J. Renouf, Howard J. Lim, Jonathan M. Loree, \\ Janine M. Davies, Sharlene Gill \\ British Columbia Cancer, Vancouver, BC, Canada \\ Contributions: (I) Conception and design: S Gill, SK Wong; (II) Administrative support: S Gill, L Gondara; (III) Provision of study materials or \\ patients: S Gill, SK Wong; (IV) Collection and assembly of data: S Gill, SK Wong; (V) Data analysis and interpretation: S Gill, L Gondara, SK \\ Wong; (VI) Manuscript writing: All authors; (VII) Final approval of manuscript: All authors. \\ Correspondence to: Sharlene Gill. 600 W 10th Ave, Vancouver, BC V5Z 4E6, Canada. Email: sgill@bccancer.bc.ca.
}

Background: Pancreatic adenocarcinoma carries a high risk of recurrence even after surgery and adjuvant chemotherapy. Current guidelines do not endorse routine surveillance imaging due to lack of evidence supporting a survival benefit. With current first-line palliative chemotherapy options, it is unclear whether surveillance allows for early detection of asymptomatic disease and therefore an improved opportunity to offer chemotherapy to fit patients. We sought to describe patterns of surveillance of resected pancreatic cancer at British Columbia (BC) Cancer and determine whether utilization of computerized tomography (CT) scans affected likelihood of receiving palliative chemotherapy at the time of recurrence.

Methods: A retrospective review was completed to identify patients treated at BC Cancer centres between 2010-2016 who had undergone curative intent resection and received at least one cycle of adjuvant chemotherapy. Information was collected on baseline characteristics, imaging scans done between adjuvant chemotherapy and recurrence, and receipt of palliative chemotherapy. Two cohorts were defined based on number of scans done between completion of adjuvant chemotherapy and recurrence: those with only 1 scan were defined as "symptomatic" recurrences and patients who had undergone more than 1 scan were considered "surveillance" recurrences.

Results: In total, 142 patients were included of which 115 (81\%) patients developed recurrence. There were 22 patients (19\%) in the "symptomatic" cohort and 93 patients (81\%) in the "surveillance" cohort. Median time to recurrence 274 days ( 9.1 months) in the symptomatic cohort compared to 471 days (15.7 months) in the surveillance group. Patients who underwent surveillance scans were more likely to receive palliative chemotherapy at the time of recurrence, though statistical significance was not reached: $51 \%$ in surveillance group versus $27 \%$ in symptomatic group [odds ratio (OR) $2.11,95 \%$ confidence interval (CI): 0.75-6.58, P=0.17].

Conclusions: Despite the absence of surveillance recommendations, the majority of patients underwent surveillance imaging. We demonstrated a non-significant increase in the likelihood of receiving palliative chemotherapy among patients who underwent surveillance scans. With more efficacious palliative chemotherapy options available, studies to determine whether receipt of chemotherapy in asymptomatic recurrences translates into improved survival and/or quality of life are warranted.

Keywords: Surveillance; pancreatic cancer; adjuvant

Submitted Oct 13, 2020. Accepted for publication Mar 05, 2021.

doi: 10.21037/jgo-20-422

View this article at: http://dx.doi.org/10.21037/jgo-20-422 


\section{Introduction}

Pancreatic ductal adenocarcinoma (PDAC) is an aggressive malignancy that is associated with significant morbidity and mortality $(1,2)$. Although the majority of patients have unresectable or metastatic disease at the time of presentation, a minority of patients present with localized, resectable disease (3). In these cases, surgical resection, often followed by adjuvant chemotherapy, remains the only potentially curative treatment option. Despite improvements in treatments, 5-year survival rates remain low at less than $10 \%$ (4). Patients are at high risk of recurrence even after surgical resection and adjuvant chemotherapy.

In other solid tumors such as colorectal cancer, postoperative surveillance is associated with earlier detection of recurrence and increased likelihood of receiving potentially curative surgery at the time of recurrent or metastatic disease, which is the basis for the recommendation of scheduled colorectal cancer surveillance in national guidelines (5-8). In contrast, guidelines for surveillance in resected pancreatic cancer are heterogeneous and the role of serial surveillance imaging remains undefined. Lack of consensus has led to significant variations in surveillance practices among providers (9-11). Even among national guidelines, discrepancies exist as recommendations are derived from expert opinion and lack high-level evidence (12). While the National Comprehensive Cancer Network (NCCN) recommends surveillance every 3-6 months for 2 years (history/physical, CA19-9, and imaging), then every 6-12 months as clinically indicated (13), the American Society of Clinical Oncology (ASCO) suggests regular visits at 3- or 6-month intervals in order to monitor for recovery of treatment-related toxicities and recurrence. The role of imaging, prolongation of surveillance intervals, and duration of surveillance are undefined (14). As for the European Society of Medical Oncology (ESMO) guidelines, they recommend no role for regular follow up after initial therapy (15).

With recent advances in available first-line palliative chemotherapy options for recurrent pancreatic cancer $(16,17)$ and recognizing that patient performance status is a key determinant of ability to receive combination chemotherapy, it is unknown whether performing surveillance imaging might allow for earlier detection of asymptomatic disease, and therefore an opportunity for patients to receive more efficacious palliative chemotherapy. In this study, we describe the patterns of surveillance in patients followed at a Canadian provincial cancer agency to determine whether imaging after adjuvant chemotherapy is associated with receipt of palliative chemotherapy upon disease recurrence. We present the following article in accordance with the STROBE reporting checklist (available at http://dx.doi.org/10.21037/jgo-20-422).

\section{Methods}

\section{Population}

British Columbia (BC) Cancer is the provincial cancer agency for the province of BC, Canada with distributed sites across the province. Following research ethics board approval, a retrospective review was completed to identify patients treated at BC Cancer centres between January 1, 2010 and December 31, 2016 who had undergone curative intent resection and received at least one cycle of adjuvant chemotherapy. Both pancreatic and periampullary cancers were included, although we hereinafter refer to the studied sample population as "pancreatic cancers". Patients were excluded if they recurred during or within 28 days after completing adjuvant chemotherapy and/or had multiple simultaneous cancer diagnoses.

\section{Data collection}

Baseline characteristics, number of imaging scans done between completing adjuvant chemotherapy and date of recurrence, and receipt of palliative chemotherapy were collected by manual chart review. Disease recurrence and date of recurrence were determined radiographically (i.e., date of first imaging study demonstrating disease recurrence/metastatic disease).

\section{Statistical analysis}

The primary endpoint of the study was estimation of the probability of receipt of palliative chemotherapy using multivariable logistic regression while adjusting for other factors such as age, sex, and the type of scan (surveillance $v s$ symptomatic). Two cohorts were defined based on number of scans done between completion of adjuvant chemotherapy and recurrence: those with only 1 scan were defined as "symptomatic" recurrences and patients who had undergone more than 1 scan were considered "surveillance" recurrences. These cohorts were established to differentiate patients who likely underwent a scan as guided by high clinical suspicion of recurrence (i.e., 


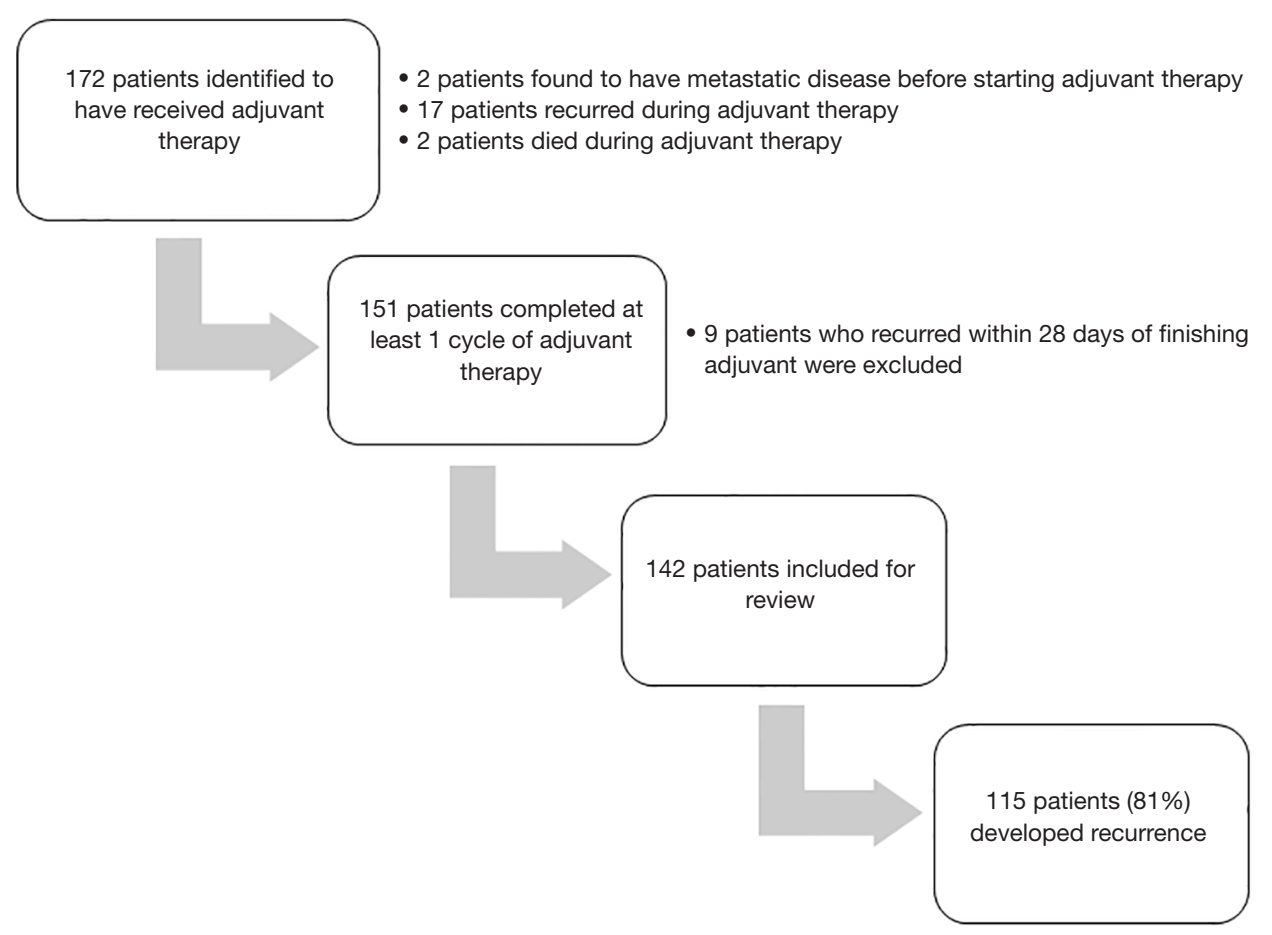

Figure 1 Flowchart of patients included in study.

presence of symptoms) versus those who underwent serial scans for reasons unrelated to new onset symptoms (i.e., surveillance). Difference in survival of the cohorts was not assessed as the variable of interest (number of scans) was confounded with survival: a patient with longer survival is likely to have more scans over the longer follow-up and a patient with aggressive disease is likely to have a higher number of scans in a short timeframe. Patient, tumour, and treatment characteristics between the cohorts were compared using the chi-square test for categorical variables and the median test for continuous variables. Statistical significance was defined as a two-sided $\mathrm{P}$ value $<0.05$. SAS 9.4 (SAS Institute, Cary, NC, USA) and R 3.5.1 was used for all statistical analysis.

\section{Ethical statement}

The study was conducted in accordance with the Declaration of Helsinki (as revised in 2013). The study was approved by University of BC Cancer Agency Research Ethics Board, H18-03243, and individual consent for this retrospective analysis was waived.

\section{Results}

A total of 142 eligible patients followed at BC Cancer were identified, of which 115 (81\%) patients had recurrence (Figure 1).

Among those with only 1 scan, defined as "symptomatic" recurrences: 22 patients (19\%), median age 68 years, 64\% female, and $91 \%$ node-positive. Within this cohort, median time to recurrence was 274 days (9.1 months) and $95 \%$ of them occurred within the first 2 years. As for patients with "surveillance" recurrences: 93 patients $(81 \%)$, median age 64 years, $43 \%$ female, $81 \%$ node-positive, median number of scans 3 . Median time to recurrence was 471 days (15.7 months) and $74 \%$ occurred within the first 2 years (Table 1).

Patients who underwent surveillance scans were more likely to receive palliative chemotherapy at the time of recurrence, but statistical significance was not reached: $51 \%$ in surveillance group versus $27 \%$ in symptomatic group [odds ratio (OR) 2.11, 95\% confidence interval (CI): 0.75$6.58, \mathrm{P}=0.17]$. Younger patients and males were more likely to receive palliative chemotherapy, although not statistically 
Table 1 Baseline patient characteristics in 115 (81\%) patients who developed recurrence: symptomatic versus surveillance cohorts. $\mathrm{P}$ values between the two cohorts for each of the baseline characteristics were done, none of which met the 0.05 level of statistical significance

\begin{tabular}{lcc}
\hline Baseline characteristics & $\begin{array}{c}\text { Symptomatic, } \\
(n=22,19 \%)\end{array}$ & $\begin{array}{c}\text { Surveillance, } \\
(n=93,81 \%)\end{array}$ \\
\hline
\end{tabular}

$\begin{array}{lcc}\text { Median age, years } & 68 & 64 \\ \text { Sex, n [\%] } & & \\ \text { Female } & 14[64] & 40[43] \\ \text { Male } & 8[36] & 53[57] \\ \text { Tumor stage, n [\%] } & & \\ \text { T1 } & 2[9] & 4[4] \\ \text { T2 } & 6[27] & 10[11] \\ \text { T3 } & 14[64] & 71[76] \\ \text { T4 } & 0[0] & 8[9]\end{array}$

Nodal status, $\mathrm{n}[\%]$

$\begin{array}{lcc}\text { No } & 2[9] & 16[17] \\ \text { N1 } & 20[91] & 75[81] \\ \text { Unknown } & 0[0] & 2[2] \\ \text { Tumor grade, n [\%] } & \\ 1 & 2[9] & 5[5] \\ 2 & 6[27] & 61[66] \\ 3 & 14[64] & 26[28] \\ \text { Unknown } & 0[0] & 1[1]\end{array}$

Resection margins, $\mathrm{n}[\%]$

Ro

$$
14[64]
$$

R1

27 [29]

$\mathrm{R} 2$

$1[5]$

Site of primary tumour, $\mathrm{n}[\%]$

$\begin{array}{lcc}\text { Head/neck } & 16[73] & 63[68] \\ \text { Body } & 5[23] & 21[23] \\ \text { Periampullary } & 1[5] & 9[10]\end{array}$

Postoperative baseline CA19-9, n [\%]

$\begin{array}{lcc}\leq 37 \text { units } / \mathrm{mL} & 12[55] & 59[63] \\ >37 \text { units } / \mathrm{mL} & 7[32] & 24[26] \\ \text { Unknown } & 3[14] & 10[11]\end{array}$

Time from surgery to recurrence, $\mathrm{n}[\%]$

$\begin{array}{lcc}<1 \text { year } & 20[91] & 26[28] \\ 1-2 \text { years } & 1[5] & 43[46] \\ >2 \text { years } & 1[5] & 24[26]\end{array}$

Table 2 Likelihood of receiving palliative chemotherapy

\begin{tabular}{lcc}
\hline Variable & OR $(95 \% \mathrm{Cl})$ & $\mathrm{P}$ value \\
\hline Older vs. younger age & $0.96(0.92-1.00)$ & 0.04 \\
Male vs. female & $2.08(0.96-4.59)$ & 0.07 \\
$\begin{array}{l}\text { Surveillance vs. symptomatic } \\
\text { recurrences }\end{array}$ & $2.11(0.75-6.58)$ & 0.17 \\
\hline
\end{tabular}

significant (Table 2).

\section{Discussion}

The rationale for surveillance is to detect recurrences early, wherein there remains a window of opportunity to offer cancer-directed interventions and improve patient outcomes. Systemic therapy, which is a cornerstone of treatment in pancreatic cancer, is a continually evolving landscape. In the curative intent setting, what was once primarily a surgical disease now warrants multi-disciplinary review and consideration of neoadjuvant and adjuvant therapies (18-20). In the palliative setting, newer treatment options have led to improvements in median overall survival $(16,17,21,22)$ and patient-reported quality of life $(23,24)$. At BC Cancer, guidelines do not recommend regular surveillance as this has not been shown to confer a survival benefit (25). In our study, the rate of recurrence among patients with resected pancreatic cancer who received at least one cycle of adjuvant chemotherapy was high at $81 \%$. Despite the absence of recommendations, the majority of patients treated with adjuvant chemotherapy for resected pancreatic cancer at BC Cancer did undergo some degree of surveillance imaging. We observed an association between imaging and a higher likelihood of receiving palliative chemotherapy ( $50 \%$ vs. $27 \%$ ). While it can be surmised that the ability to receive chemotherapy would be associated with an improvement in patient outcomes, larger studies would be required to confirm this and determine whether earlier receipt of chemotherapy in surveillance-detected recurrences results in improvements in survival and quality of life.

Similar to our study investigating likelihood of receiving palliative chemotherapy, Daamen et al. undertook a recent Netherlands-based observational cohort study of 836 resected PDAC patients, investigating whether early detection of asymptomatic PDAC recurrence increased likelihood of receiving additional treatment and if receipt of additional treatments translated into improved survival 
rates (26). Out of $670(80 \%)$ patients who developed recurrence, $76 \%$ were symptomatic and $24 \%$ were asymptomatic. Thirty-one percent of symptomatic recurrences received additional treatment (chemotherapy, radiation, or other therapies) compared to $48 \%$ of their asymptomatic counterparts. Additional treatment of PDAC recurrence was independently associated with improved OS for both symptomatic and asymptomatic patients: hazard ratio (HR) 0.53 , 95\% CI: $0.42-0.67, \mathrm{P}<0.001$ and HR 0.45 , 95\% CI: $0.29-0.70, \mathrm{P}<0.001$, respectively.

Numerous other studies have been done to elucidate the relationship between surveillance and survival benefit. These have yielded mixed results and should be interpreted cautiously as there have been ongoing improvements in palliative chemotherapy regimens. A retrospective review of patients with resected ductal adenocarcinoma between 2000 and 2013 was done by $\mathrm{Wu}$ et al., investigating differences in median overall survival between four different surveillance strategy groups: symptom group, imaging group, tumor marker group, and intense group (history/ physical, tumor markers, and imaging). They did not find any statistical difference between the four subgroups, albeit the authors also acknowledged that more recent advances in palliative chemotherapy, such as FOLFIRINOX, and advances in treatments for local recurrences may not have been encompassed by their patient sample (27). Similarly, Witkowski et al. quantified nationwide use of imaging after pancreatic cancer resection and found a doubling in the median number of scans done between 1991 and 2005, but no associated survival benefit (28).

Others have found that surveillance may lead to higher rates of detecting recurrences and improved overall survival. A retrospective review presented as an abstract in 2010 by Shabahang et al. compared three groups of patients who underwent different surveillance regimens. Recommended follow-up was defined as serial carbohydrate antigen 19-9 (CA19-9) and computerized tomography (CT) imaging every 3 months in the first year, every 6 months in the second year, and annually thereafter. One group underwent the follow-up as recommended [ $\mathrm{n}=19(14 \%)]$, one group had follow-up but not as recommended $[\mathrm{n}=82(59 \%)]$, and finally the last group had elected not to undergo routine follow-up [ $\mathrm{n}=38(27 \%)]$. The median survival periods for the 3 groups were $16.6,15.7$, and 8.7 months, respectively. The authors' conclusions were in support of a scheduled surveillance program (29).

Although we did not collect information regarding the absence or presence of symptoms at the time of recurrence in our study, one hypothesis why patients who undergo scans may be more likely to receive subsequent palliative chemotherapy is the detection of recurrences while patients are still asymptomatic and clinically fit. Often what precludes physicians from offering chemotherapy to patients, apart from pre-existing medical comorbidities, is their performance status. In a study by Nordby et al., 28\% of asymptomatic recurrences received best supportive care only compared to $63 \%$ of symptomatic patients (30). Not only are asymptomatic patients more likely to receive oncologic treatments at the time of recurrence compared to their symptomatic counterparts, but early detection could make the difference between a patient being able to tolerate a triplet chemotherapy regimen such as FOLFIRINOX as opposed to single agent regimens. Tzeng et al. also described significantly longer median overall survival in asymptomatic recurrences (29.6 vs. 18.0 months). Asymptomatic patients were more likely to receive treatment $(91.2 \%$ vs. $61.4 \%)$ at the time of recurrence which translated to a significantly longer postresection overall survival (11.8 vs. 2.6 months, $\mathrm{P}<0.001)$ (31).

Apart from the opportunity to offer systemic treatments, there may still be a role for early detection of recurrence. At the time of recurrence, preserved performance status and isolated recurrence (local or distant as opposed to regional or multiple-site recurrence) are independently associated with longer post-resection overall survival (31). Symptomatic recurrence, on the other hand, is an independent predictor of poor post-recurrence survival (30). Early detection of limited, locoregional recurrence may also offer the opportunity for surgical interventions. Up to one third of pancreatic cancer recurrences are isolated local recurrences (32-35), and surgical resection could confer survival benefit (36). A study by Tjaden et al. demonstrates how structured detection of recurrences facilitates the ability to offer subsequent treatments. In their cohort, they implemented structured, postoperative follow-up after surgery for PDAC: CT scans 3 -monthly for 2 years, followed by 6-monthly afterwards. They found that not only was the presence of symptoms an unreliable indicator of recurrence (only $26 \%$ of patients had reported symptoms at the time of recurrence), but also that all 74 out 184 (40\%) PDAC patients who recurred went on to receive cancer-directed treatment including 11 of 16 patients who underwent re-resection for local recurrence (37).

Ultimately, consensus regarding the role of surveillance and development of a structured surveillance strategy is 
important in the setting of resected pancreatic cancer. Studies investigating surveillance that were done prior to the development of newer palliative chemotherapy regimens may not reflect the full extent of benefits to detecting early, asymptomatic recurrences and the ability to offer subsequent efficacious therapies or opportunity to participate in clinical trials. The development of a surveillance schedule requires multiple considerations including cost-effectiveness (38), modality of surveillance, frequency, and duration. CA199 has been previously demonstrated to be predictive of survival (27,39-41), and the observation of its rise preceding detection of recurrence by imaging (42) may be helpful as a more cost-effective surveillance modality than imaging alone. Similar to previous studies which have found the highest risk of recurrence 2 years after surgery $(43,44), 78 \%$ of recurrences occurred within 2 years in our study. Median time to recurrence was 9.1 months in the symptomatic cohort and 15.7 months in the surveillance cohort. Implementing a surveillance schedule with the combination of blood work and imaging over 2 years postoperatively wherein patients are at highest risk of recurrence would offer a balance between cost and benefit. The role of primary care practitioners in providing follow up after surgical resection and completion of adjuvant chemotherapy is also an important consideration in generating a consensus for surveillance (45-48).

The limitations of our study include those inherent to its retrospective design. Given the non-randomized, retrospective nature of this study, it is difficult to discern whether differences in ability to receive palliative chemotherapy between symptomatic and surveillance patients may also be related to differences in disease biology. However, our findings are consistent with the recent Netherlands-based observational cohort study (26). Our sample size was relatively small and limited to patients treated at one Canadian provincial cancer agency (across six BC Cancer sites). We were unable to determine the indications for all scans done postoperatively nor were we able to determine the presence or absence of symptoms or CA19-9 levels at the time of recurrence. We did not evaluate other patient factors such as preference, tolerance of adjuvant chemotherapy, and performance status upon completion of adjuvant therapy, all of which are clinical considerations in determining whether or not surveillance may be appropriate. Finally, we also acknowledge that despite the trend towards increased likelihood of receiving palliative chemotherapy in patients found to have recurrence through surveillance scans, we are unable to determine whether or not receipt of palliative chemotherapy translates into improved outcomes.

To conclude, our study demonstrates that the majority of patients treated in BC underwent some form of surveillance imaging after the completion of surgery and adjuvant chemotherapy. Within the limits of our sample size, we demonstrate a trend towards increased likelihood of receiving palliative chemotherapy in patients found to have recurrence through surveillance as opposed to new onset symptoms. With the evolution of more efficacious palliative chemotherapy regimens, prospective studies to determine whether receipt of palliative chemotherapy in asymptomatic recurrences detected on imaging translates into improved survival and/or quality of life are warranted. The psychological impact of undergoing surveillance on patients' perception of care and anxiety regarding recurrence should also be evaluated.

\section{Acknowledgments}

The study was presented at Gastrointestinal Cancers Symposium 2020 and published as an online abstract at ASCO 2020.

Funding: None.

\section{Footnote}

Reporting Checklist: The authors have completed the STROBE reporting checklist. Available at http://dx.doi. org/10.21037/jgo-20-422

Data Sharing Statement: Available at http://dx.doi. org/10.21037/jgo-20-422

Conflicts of Interest: All authors have completed the ICMJE uniform disclosure form (available at http://dx.doi. org/10.21037/jgo-20-422). DJR reports grants and personal fees from Roche, and personal fees from Celgene, Servier, Ipsen, Taiho, Bayer, and Astra Zeneca, all outside the submitted work. HJL reports serving on advisory boards for Ipsen, Roche, Amgen, Eisai, Taiho, and BMS, all outside the submitted work. JML reports grants and personal fees from Amgen, grants and personal fees from Ipsen, and personal fees from Novartis, Eisai, Bayer, and Pfizer, all outside the submitted work. JMD reports personal fees from Novartis and Astra Zeneca, outside the submitted work. The other 
authors have no conflicts of interest to declare.

Ethical Statement: The authors are accountable for all aspects of the work in ensuring that questions related to the accuracy or integrity of any part of the work are appropriately investigated and resolved. The study was conducted in accordance with the Declaration of Helsinki (as revised in 2013). The study was approved by University of British Columbia Cancer Agency Research Ethics Board, H18-03243, and individual consent for this retrospective analysis was waived.

Open Access Statement: This is an Open Access article distributed in accordance with the Creative Commons Attribution-NonCommercial-NoDerivs 4.0 International License (CC BY-NC-ND 4.0), which permits the noncommercial replication and distribution of the article with the strict proviso that no changes or edits are made and the original work is properly cited (including links to both the formal publication through the relevant DOI and the license). See: https://creativecommons.org/licenses/by-nc-nd/4.0/.

\section{References}

1. Gupta R, Amanam I, Chung V. Current and future therapies for advanced pancreatic cancer. J Surg Oncol 2017;116:25-34.

2. Hidalgo M. Pancreatic cancer. N Engl J Med 2010;362:1605-17.

3. Katz MH, Wang H, Fleming JB, et al. Long-term survival after multidisciplinary management of resected pancreatic adenocarcinoma. Ann Surg Oncol 2009;16:836-47.

4. Siegel RL, Miller KD. Cancer statistics, 2019. CA Cancer J Clin 2019;69:7-34.

5. Massaut E, Bohlok A, Lucidi V, et al. The concept of oligometastases in colorectal cancer: from the clinical evidences to new therapeutic strategies. Curr Opin Oncol 2018;30:262-8.

6. Costas-Chavarri A, Nandakumar G, Temin S, et al. Treatment of Patients With Early-Stage Colorectal Cancer: ASCO Resource-Stratified Guideline. J Glob Oncol 2019;5:1-19.

7. Desch CE, III ABB, Somerfield MR, et al. Colorectal Cancer Surveillance: 2005 Update of an American Society of Clinical Oncology Practice Guideline. J Clin Oncol 2005;23:8512-9.

8. (NCCN) NCG. NCCN Clinical Practice Guidelines in Oncology: Colon Cancer. Version 2.2021. 2021. Available online: https://www.nccn.org/professionals/physician_ gls/pdf/colon.pdf

9. Castellanos JA, Merchant NB. Intensity of followup after pancreatic cancer resection. Ann Surg Oncol 2014;21:747-51.

10. O'Reilly EM, Lowery MA. Postresection surveillance for pancreatic cancer performance status, imaging, and serum markers. Cancer J 2012;18:609-13.

11. Sheffield KM, Crowell KT, Lin YL, et al. Surveillance of pancreatic cancer patients after surgical resection. Ann Surg Oncol 2012;19:1670-7.

12. Poonacha TK, Go RS. Level of scientific evidence underlying recommendations arising from the National Comprehensive Cancer Network clinical practice guidelines. J Clin Oncol 2011;29:186-91.

13. National Comprehensive Cancer Network (NCCN) Clinical Practice Guidelines; Pancreatic Adenocarcinoma. V.1.2020. Available online: https://www.nccn.org/ professionals/physician_gls/default.aspx\#pancreatic. Accessed June 4, 2020.

14. Khorana AA, McKernin SE, Berlin J, et al. Potentially Curable Pancreatic Adenocarcinoma: ASCO Clinical Practice Guideline Update. J Clin Oncol 2019;37:2082-8.

15. Ducreux M, Cuhna AS, Caramella C, et al. Cancer of the pancreas: ESMO Clinical Practice Guidelines for diagnosis, treatment and follow-up. Ann Oncol 2015;26 Suppl 5:v56-68.

16. Conroy T, Desseigne F, Ychou M, et al. FOLFIRINOX versus gemcitabine for metastatic pancreatic cancer. $\mathrm{N}$ Engl J Med 2011;364:1817-25.

17. Von Hoff DD, Ervin T, Arena FP, et al. Increased survival in pancreatic cancer with nab-paclitaxel plus gemcitabine. N Engl J Med 2013;369:1691-703.

18. Conroy T, Hammel P, Hebbar M, et al. FOLFIRINOX or Gemcitabine as Adjuvant Therapy for Pancreatic Cancer. N Engl J Med 2018;379:2395-406.

19. Neoptolemos JP, Palmer DH, Ghaneh P, et al. Comparison of adjuvant gemcitabine and capecitabine with gemcitabine monotherapy in patients with resected pancreatic cancer (ESPAC-4): a multicentre, open-label, randomised, phase 3 trial. Lancet 2017;389:1011-24.

20. Oettle H, Post S, Neuhaus P, et al. Adjuvant chemotherapy with gemcitabine vs observation in patients undergoing curative-intent resection of pancreatic cancer: a randomized controlled trial. JAMA 2007;297:267-77.

21. Wang-Gillam A, Hubner RA, Siveke JT, et al. NAPOLI-1 phase 3 study of liposomal irinotecan in metastatic pancreatic cancer: Final overall survival analysis and 
characteristics of long-term survivors. Eur J Cancer 2019;108:78-87.

22. Wang-Gillam A, Li CP, Bodoky G, et al. Nanoliposomal irinotecan with fluorouracil and folinic acid in metastatic pancreatic cancer after previous gemcitabine-based therapy (NAPOLI-1): a global, randomised, open-label, phase 3 trial. Lancet 2016;387:545-57.

23. Gourgou-Bourgade S, Bascoul-Mollevi C, Desseigne F, et al. Impact of FOLFIRINOX compared with gemcitabine on quality of life in patients with metastatic pancreatic cancer: results from the PRODIGE 4/ACCORD 11 randomized trial. J Clin Oncol 2013;31:23-9.

24. Picozzi V, Narayanan S, Henry Hu X, et al. HealthRelated Quality of Life in Patients with Metastatic Pancreatic Cancer. J Gastrointest Cancer 2017;48:103-9.

25. British Columbia Cancer Agency Cancer Management Guidelines; Gastrointestinal Cancer; Pancreas. Available online: http://www.bccancer.bc.ca/health-professionals/ clinical-resources/cancer-management-guidelines/ gastrointestinal/pancreas. Accessed June 4, 2020.

26. Daamen LA, Groot VP, Besselink MG, et al. Detection, Treatment, and Survival of Pancreatic Cancer Recurrence in the Netherlands: A Nationwide Analysis. Ann Surg 2020. [Epub online ahead of print]. doi: 10.1097/ SLA.0000000000004093.

27. Wu H, Guo JC, Yang SH, et al. Postoperative Imaging and Tumor Marker Surveillance in Resected Pancreatic Cancer. J Clin Med 2019;8:1115.

28. Witkowski ER, Smith JK, Ragulin-Coyne E, et al. Is it worth looking? Abdominal imaging after pancreatic cancer resection: a national study. J Gastrointest Surg 2012;16:121-8.

29. Shabahang M, Marchessault M, Luh J. The effect of close postoperative follow-up on survival outcome of patients with pancreatic cancer. 2010 Gastrointestinal Cancers Symposium. Vol. 262. 2010.

30. Nordby T, Hugenschmidt H, Fagerland MW, et al. Follow-up after curative surgery for pancreatic ductal adenocarcinoma: asymptomatic recurrence is associated with improved survival. Eur J Surg Oncol 2013;39:559-66.

31. Tzeng CW, Fleming JB, Lee JE, et al. Yield of clinical and radiographic surveillance in patients with resected pancreatic adenocarcinoma following multimodal therapy. HPB (Oxford) 2012;14:365-72.

32. Hishinuma S, Ogata Y, Tomikawa M, et al. Patterns of recurrence after curative resection of pancreatic cancer, based on autopsy findings. J Gastrointest Surg 2006; 10:511-8.
33. Neoptolemos JP, Stocken DD, Friess H, et al. A randomized trial of chemoradiotherapy and chemotherapy after resection of pancreatic cancer. N Engl J Med 2004;350:1200-10.

34. Raut CP, Tseng JF, Sun CC, et al. Impact of resection status on pattern of failure and survival after pancreaticoduodenectomy for pancreatic adenocarcinoma. Ann Surg 2007;246:52-60.

35. Zhang Y, Frampton AE, Kyriakides C, et al. Locorecurrence after resection for ductal adenocarcinoma of the pancreas: predictors and implications for adjuvant chemoradiotherapy. J Cancer Res Clin Oncol 2012;138:1063-71.

36. Strobel O, Hartwig W, Hackert T, et al. Re-resection for isolated local recurrence of pancreatic cancer is feasible, safe, and associated with encouraging survival. Ann Surg Oncol 2013;20:964-72.

37. Tjaden C, Michalski CW, Strobel O, et al. Clinical Impact of Structured Follow-up After Pancreatic Surgery. Pancreas 2016;45:895-9.

38. Tzeng CW, Abbott DE, Cantor SB, et al. Frequency and intensity of postoperative surveillance after curative treatment of pancreatic cancer: a cost-effectiveness analysis. Ann Surg Oncol 2013;20:2197-203.

39. Abdel-Misih SR, Hatzaras I, Schmidt C, et al. Failure of normalization of CA19-9 following resection for pancreatic cancer is tantamount to metastatic disease. Ann Surg Oncol 2011;18:1116-21.

40. Ferrone CR, Finkelstein DM, Thayer SP, et al. Perioperative CA19-9 levels can predict stage and survival in patients with resectable pancreatic adenocarcinoma. $\mathrm{J}$ Clin Oncol 2006;24:2897-902.

41. Humphris JL, Chang DK, Johns AL, et al. The prognostic and predictive value of serum CA19.9 in pancreatic cancer. Ann Oncol 2012;23:1713-22.

42. Rieser CJ, Zenati M, Hamad A, et al. CA19-9 on Postoperative Surveillance in Pancreatic Ductal Adenocarcinoma: Predicting Recurrence and Changing Prognosis over Time. Ann Surg Oncol 2018;25:3483-91.

43. Kovač JD, Mayer P, Hackert T, et al. The Time to and Type of Pancreatic Cancer Recurrence after Surgical Resection: Is Prediction Possible? Acad Radiol 2019;26:775-81.

44. Van den Broeck A, Sergeant G, Ectors N, et al. Patterns of recurrence after curative resection of pancreatic ductal adenocarcinoma. Eur J Surg Oncol 2009;35:600-4.

45. Grunfeld E, Gray A, Mant D, et al. Follow-up of breast cancer in primary care vs specialist care: results of an 
economic evaluation. Br J Cancer 1999;79:1227-33.

46. Grunfeld E, Levine MN, Julian JA, et al. Randomized trial of long-term follow-up for early-stage breast cancer: a comparison of family physician versus specialist care. J Clin Oncol 2006;24:848-55.

47. Samawi HH, Yin Y, Lim HJ, et al. Primary Care Versus Oncology-Based Surveillance Following Adjuvant

Cite this article as: Wong SK, Gondara L, Renouf DJ, Lim HJ, Loree JM, Davies JM, Gill S. Impact of surveillance among patients with resected pancreatic cancer following adjuvant chemotherapy. J Gastrointest Oncol 2021;12(2):446454. doi: 10.21037/jgo-20-422
Chemotherapy in Resected Pancreatic Cancer. J

Gastrointest Cancer 2018;49:429-36.

48. Wattchow DA, Weller DP, Esterman A, et al. General practice vs surgical-based follow-up for patients with colon cancer: randomised controlled trial. Br J Cancer 2006;94:1116-21. 